\title{
SVM-BetaPred: Prediction of Right-Handed B-Helix Fold from Protein Sequence Using SVM
}

\author{
Siddharth Singh ${ }^{1}$, Krishnan Hajela ${ }^{2}$, and Ashwini Kumar Ramani ${ }^{1}$ \\ ${ }^{1}$ School of Computer Science \\ ${ }^{2}$ School of Life Science, Devi Ahilya University, \\ Khandwa Road, Indore-452001, India \\ \{sidssingh, hajelak\}@gmail.com, ramani.iips@dauniv.ac.in
}

\begin{abstract}
The right-handed single-stranded B-helix proteins are characterized as virulence factors, allergens, toxins that are threat to human health. Identification of these proteins from amino acid sequence is of great importance as these proteins are potential targets for anti-bacterial and fungal agents. In this paper, support vector machine (SVM) has been used to predict the presence of B-helix fold in protein sequences using dipeptide composition. An input vector of 400 dimensions for dipeptide compositions is used to search for the presence of putative rungs or coils, the conserved secondary structure, found in $\beta$-helix proteins. An average accuracy of $89.2 \%$ with Matthew's correlation coefficient of 0.75 is obtained in a 5 -fold cross-validation technique. In addition, a PSSM was also used to score the query sequence of proteins identified as B-helices by SVM. The method recognizes right-handed B-helices with $100 \%$ sensitivity and $99.6 \%$ specificity on test set of known protein structures.
\end{abstract}

Keywords: ß-helix fold, ß-sheet stacking, pectate lyase, fold recognition, secondary structure, Support Vector Machines, SVM, PSSM.

\section{Introduction}

The right-handed single-stranded parallel $\beta$-helices $[\mathrm{R} \beta \mathrm{H}]$ are perfect examples of protein fold that display high three-dimensional structural similarity in spite of low sequence homologies across the families [1]. These proteins display diverse functions ranging from the enzymatic activities to specific structural roles [2,3]. The characteristic feature of these proteins is the presence of a coiled polypeptide backbone having almost same circular arrangement of $\beta$-sheet structure elements in space $[1,4,5]$. The backbone alternates between $\beta$-strands and turns with the $\beta$-sheets running perpendicular to the axis of protein molecule as shown in Figure 1. As a result of this progressive coiling of backbone, the buried hydrophobic cores of B-helix domains is extended, rather than globular, and is characterized by the distinctive stacking of side chains that occur at equivalent position in successive coils called rungs or turns. A comparative analysis of different known $\beta$-helices has revealed strong positional preference for specific amino acid residues towards the interior of parallel $\beta$-helices [6,7]. The $\beta$-helix fold lacks an obvious sequence repeats, although presence of some sequence patterns has been reported across the super-families $[3,8]$. 
In recent past, a shift from classical fold recognition approach to machine learning has been observed. For example, Leslie et al [9], have developed a sequence similarity based kernel for protein fold classification. Cheng et al [10], present a good overview of these fold recognition methods. A number of attempts have been made to predict the B-helix fold across the family of known structure. Heffron et al [3] developed a sequence profile based on pectate lyase family under SCOP classification [11], however, the method could only predict few proteins from pectate lyase and pectin lyase families as $\mathrm{R} ß \mathrm{H}$ proteins. Other general fold prediction methods like PSIBLAST [12] and HMMer [13], fail to identify sequences across the RßH fold $[1,14]$. BetaWrap, which depends mainly on $\beta$-strand interactions, shows some success in predicting correct fold across the families in the $\mathrm{R} \beta \mathrm{H}$ fold. However, the method when tested on Swiss-Prot \& TrEmBL [15] predicts a number of LRR, L $\beta H$ and other structurally related proteins as right-handed B-helix proteins. Immunoglobulin-like Bsandwiches, TIM beta/alpha barrels, the FAD-NAD(P)-binding domain, acid proteases, the subtilisin-like fold and various beta-propeller motifs also appear as false positives [1]. Although these protein classes bear less structural homology with B-helix proteins, many of these contain amphiphatic $B$-sheets that are highly favored by BetaWrap algorithm. Recently, threading has emerged as a very successful method for the prediction of protein folds and structure across the parallel B-helix fold [16].

In this paper, a systematic attempt has been made to achieve high prediction accuracy for B-helix fold from proteins sequences. A SVM module, SVM-BetaPred, is developed based on dipeptide compositions (e.g. ala-ala, ala-leu, val-ser) of known B-helix proteins to correctly identify rungs or coils in protein sequence. These rungs are the characteristic feature of $\beta$-helix proteins, each displaying a conserved B2-T2-B3 pattern. The initial screening indicates that the prediction accuracy of the dipeptide composition based SVM module is superior to the amino acid
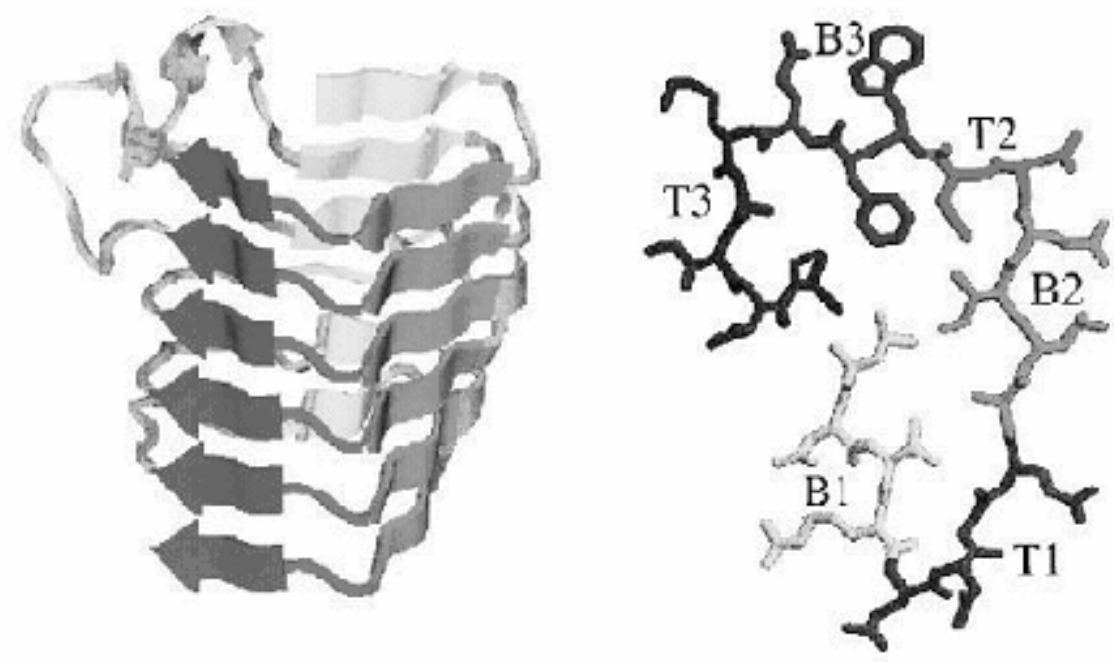

Fig. 1. Ribbon diagram of right-handed single-stranded B-helix protein (1plu) and schematic representation of a single rung or coil [1] 
composition and tripeptide composition based modules. In addition, a position specific scoring matrix (PSSM) is constructed using multiple sequence alignments of known B-helix proteins in SCOP database. This PSSM is used to calculate a log odds score for each putative rung identified by the dipeptide SVM module. The predicted rungs are wrapped into a $\beta$-helix fold using a number of rules learned from known $\beta$ helices. Finally, a summed score of all the putative rungs identified in a protein is used as a threshold cut off to predict the $\beta$-helix proteins. The method performs at par with some of the state-of-art methods for $\beta$-helix fold prediction.

\section{Materials and Methods}

\subsection{Training and Testing Data Set}

All positive and negative datasets for training and testing SVM modules are derived from B-helix database that consists of right-handed single-stranded proteins in SCOP 1.69 database. The RßH fold consists of seven superfamilies, however, insect antifreeze proteins (AFPs) are not considered in this study, as their sequence and structure patterns do not represent an ideal $\beta$-helix fold. The method, however, successfully predicts AFPs as $B$-helix proteins. The B-helix database is represented by 28 unique sequences. The SCOP structures are used to annotate the rungs in all proteins. The positive data set consists of 253 rungs of length nine amino acids that are derived from the known $\beta$-helices. The negative data set consists of 261 loop regions of length nine derived from the known $\beta$-helix database. The SVM module is trained with various window size that range from 9 to 15 .

\subsection{Evaluation Data Set}

The negative $\beta$-helix protein dataset, PDB-minus, is composed of 5049 non- $\beta$-helical proteins sequences with no sequences having homology more than $33 \%$ is used. This dataset can be downloaded from the following website: http://cubic.bioc.columbia.edu/ eva/res/unique_list.html. All known $\beta$-helix proteins were removed for this dataset.

\subsection{Prediction Data Set}

Potential new B-helices are identified by SVM-BetaPred from the UniProtKB/SwissProt Release 51.2 of 28-Nov-2006 with 243975 sequence entries. This database is filtered to eliminate sequence with more than $40 \%$ sequence homology. The resulting dataset, SW40 dataset, contains 67,879 sequences. Incremental redundancy filtering is accomplished using the CD-HIT program [17]. All sequences of length less than 100 amino acids are eliminated from SW40 dataset, as ideal B-helix proteins cannot be less than 100 amino acids in length.

\subsection{Support Vector Machine}

Support Vector Machines [SVM] are universal approximators based on statistical and optimization theory [18,19]. A Support Vector Machine (SVM) performs classification by constructing a $\mathrm{N}$-dimensional hyperplane that optimally separates the 
data into two categories. SVMs have emerged as a high performance tool to solve classification problems. A number of problems in computational biology are classification problems and SVM can be applied to these problems effectively due to its ability to handle noise, large dataset and large input spaces [18,19]. Vapnik et al [20] have described SVM in details. In the current studies, SVMlight is used to predict putative rungs in a protein sequence. This software is freely downloadable from http://svmlight.joachims.org. SVMlight is an implementation of Vapnik's Support Vector Machine [21] for the problem of pattern recognition, regression and learning a ranking function. The software enables the users to define a number of parameters and also allows a choice of inbuilt kernel function, including linear, RBF and Polynomial.

We have developed a dipeptide composition based binary classifier to handle the rung prediction problem. The problem of right-handed $\beta$-helix fold prediction can be considered as a classification problem. The main aim is to predict the presence of rungs in a given protein sequence. In this classification problem a rung can be considered as an object $\vec{x}$, which is a part of protein amino acids sequence. A class +1 can be assigned to this object if the center of $\vec{x}$ lies inside a rung or -1 otherwise.

\subsection{SVM Features}

Amino acid, dipeptide and tripeptide frequency are used to train RBF kernels at default parameters, the accuracies are $72.1 \%, 89.3 \%$ and $78.9 \%$ respectively. These frequencies are obtained from the positive rungs and negative loop regions discussed above. The frequencies are obtained using the following equation:

$$
\text { Frequency of } k-\text { mer }=\frac{\text { Total number of } k-\text { mer }}{\text { Total number of all possible } k-\text { mers }}
$$

where $\mathrm{k}=1,2$ and 3 which give a fixed length pattern of 20, 20x20 and 20x20x20 respectively. SVM accuracy was determined to be highest for dipeptide frequency thus all further training and predictions were restricted to dipeptide frequency. Some of the important dipeptides reported are NV, SG, VT, TI, IT, NI, TV, GA, GG, DV, NS, VI.

\subsection{Kernel and Parameters}

The selection of the kernel function parameters is an important step for SVM training and testing. All types of kernels i.e. RBF, Polynomial, Sigmoid and Linear were tested, and RBF kernel was identified to have the best performance for rung prediction. A number of parameters need to be determined to generate optimal results, the most important of these are the regularization parameter $\mathrm{C}$ and $\gamma$ for RBF kernel. The prediction accuracy of various parameter values is shown in Table 1. The dipeptide based SVM modules with RBF kernel, $\mathrm{C}=18$ and $\gamma=900$ is used for training. 
Table 1. Prediction accuracy comparison for different parameters for RBF kernel function

\begin{tabular}{|c|c|c|}
\hline SVM Model & Parameters & Accuracy (\%) \\
\hline 1 & $\mathrm{C}=$ default, $\gamma=$ default & 89.93 \\
\hline 2 & $\mathrm{C}=0.1, \gamma=1000$ & 96.51 \\
\hline 3 & $\mathrm{C}=1, \gamma=1000$ & 97.83 \\
\hline 4 & $\mathrm{C}=10, \gamma=1000$ & 98.49 \\
\hline 5 & $\mathrm{C}=18, \gamma=900$ & 98.82 \\
\hline 6 & $\mathrm{C}=24, \gamma=900$ & 98.13 \\
\hline
\end{tabular}

\subsection{SVM Input}

Increasing the window size can provide more local sequence information. The window size $\mathrm{w}$ is defined as the residue numbers involved in the local sequence windows centered on fifth residue of a rung of length 9, i.e. $\mathrm{w}=9,10,11,12,13,14$ and 15 in this study. Different local window sizes are used to build the SVM models in order to find one, which could lead to the best performance. The prediction accuracy is shown in Table 2. As expected, the overall prediction accuracy Q2 [22] increases with the enlarging window size and attain its peak at 13. As window size increases so does the background noise while smaller window size results in less useful information. Accordingly, the optimal window size is fixed to 13 for further analysis in this study. The rungs are extended both upstream and downstream to obtain different window size.

Table 2. Predictive performance of SVM based on single sequence inputs of different local window sizes

\begin{tabular}{|c|c|c|c|c|}
\hline $\begin{array}{c}\text { Window } \\
\text { Size }\end{array}$ & \multicolumn{4}{|c|}{ Prediction accuracy (\%) } \\
\hline & Sensitivity & Specificity & $\mathrm{Q}_{2}$ & MCC \\
\hline 9 & 52.8 & 94.8 & 81.7 & 0.54 \\
\hline 10 & 52.8 & 95.6 & 82.2 & 0.56 \\
\hline 11 & 54.7 & 96.5 & 83.4 & 0.60 \\
\hline 12 & 62.2 & 98.3 & 86.9 & 0.69 \\
\hline 13 & 64.2 & 100.0 & 88.7 & 0.74 \\
\hline 14 & 60.3 & 99.1 & 86.9 & 0.70 \\
\hline 15 & 60.3 & 97.4 & 85.8 & 0.66 \\
\hline
\end{tabular}

\subsection{SVM Output}

The output from SVM light is in form of real number, a positive value denotes a rung while a negative value denotes a loop region.

\subsection{Performance Evaluation}

To evaluate the prediction performance of the SVM module, a 5-fold cross-validation method is used. The dataset were randomly divided into five groups, with each group 
containing roughly equal numbers of proteins. Each group was singled out in turns as the testing dataset, while the remaining proteins in other groups were used as the training dataset. Four different measurements have been used to measure the prediction performance of SVM module [22], these are:

$$
\text { sensitivity }=\frac{T P}{T P+F N}
$$

where, TP is the number of true positives and $\mathrm{FN}$ is the number of false negatives or under-predictions.

$$
\text { specificity }=\frac{T N}{T N+F P}
$$

where, $\mathrm{TN}$ is the number of true negatives, and FP is the number of false positives or over-predictions.

The overall prediction accuracy $\mathrm{Q}_{2}$ is given by

$$
Q_{2}=\frac{T P+T N}{T P+T N+F P+F N}
$$

The Matthews Correlation Coefficient (MCC) [23] is given by

$$
M C C=\frac{T P \times T N-F P \times F N}{\sqrt{(T P+F P)(T P+F N)(T N+F P)(T N+F N)}}
$$

The value of MCC is 0 for a random assignment and 1.0 for a perfect prediction.

\subsection{Position Specific Scoring Matrix}

All rungs of size 13 were extracted from the $\beta$-helices proteins available in SCOP database. These rungs were used to generate a position specific weight matrix. The main aim is to score the putative rungs identified by the dipeptide frequency based SVM module. The number of occurrences of each amino acid at a given position is compiled. Prophecy module in EMBOSS package [24] is used to generate the frequency matrix for amino acid occurrence at a specific position in the rungs. These counts are converted to frequencies that are used to calculate log odds scores. The odds score [25] is the frequency observed divided by the theoretical frequency expected (the background frequency of the amino acid, usually averaged over the protein $\sim 0.05 /$ aminoacid). For example, if the amino acid frequency is 0.79 and the estimated background frequency is 0.25 , the odds score would be $0.79 / 0.25=3.16$. Finally, odds scores were converted to log odds scores by taking the logarithm base 2 .

$$
W_{i . j}=\log _{2}\left(\frac{F_{i, j}}{P_{i}}\right)
$$


where $W_{i . j}$ is the scoring matrix value of amino acid $i$ in position $j . F_{i, j}$ is the frequency of base $i$ in position $j$ and $\mathrm{P}_{i}$ is the background frequency of base $i$.

As the logarithm of zero is infinity, a zero occurrence of a particular base in the matrix creates a problem. In this case, a very low frequency value of 0.0001 is assigned to amino acid count at such a position in a scoring matrix. PSSM is available as supplementary material at http://www.scs.dauniv.ac.in/research.php.

\section{The Algorithm}

\subsection{Rung Prediction in Protein Sequence}

The dipeptide composition based SVM module as described above is used to predict rungs in protein sequences. A window of size 13 is used to predict rungs across a protein sequence. All predictions with score of 1 or above are selected as valid rungs. This cutoff was derived from known $\beta$-helix proteins. These putative rungs are wrapped into $\beta$-helix using a minimum distance threshold of 17 and a maximum distance threshold of 70 amino acids between adjacent rungs. In case, if two adjacent rungs are closer than the minimum distance threshold, then the rung with higher score is selected. Similarly, if the distance between two adjacent rungs is more than the maximum allowed distance and number of valid rungs is less than 5, then valid rungs counter is reset to zero and the existing rungs are rejected. The process is repeated for the rest of the protein sequence till at least a single wrap of 5 valid rungs is obtained. Proteins with a wrap of less than 5 rungs are rejected. Once the wraps are generated they are filtered for the presence of charged residues in rungs. This filter assigns a heavy demerit to rungs for presence of highly charged residues at inward pointing positions in rungs.

\subsection{Log Odds Score for Wrap}

A log odds score is determined for every predicted wrap using the PSSM. All adjacent rungs are aligned to generate a pairwise log odds score. The PSSM is used to refer the frequencies of occurrence of amino acid $\mathrm{X}$ at a position while calculating the score of aligned rung pair. A substitution score is calculated for each position in the two adjacent rungs, for example, log odds score is calculated for occurrence of amino acid $\mathrm{X}$ at position 1 in rung $\mathrm{A}$ as to the occurrence of amino acid $\mathrm{Y}$ at the position 1 in rung $\mathrm{B}$. The $\log$ ratio for all the thirteen position of rungs is summed to give a pairwise log odds score. A random score of -1000 is assigned to alignment of a rung with itself. Once a pairwise log odds score is calculated for all the adjacent rung pairs, a $\log$ odds score for a wrap is calculated by taking the sum of pairwise log odds scores. All proteins with a wrap log odds score of -35 or more are further processed. The log odds score derived using PSSM helps in filtering a number of proteins that appear as false positives in SVM predictions, for example, LRR and amphiphatic proteins that have amino acids composition similar to $\beta$-proteins. 


\subsection{Completing the Parse}

Now that the position of rungs have been determined using SVM module, the position of B1 strands are determined using PSIPRED [26] predictions. Small $\beta$-sheets of average length four amino acids cannot be effectively predicted by SVM module, thus the secondary structure predictions from PSIPRED are used to place B1 strands between adjacent B2-T2-B3 rungs. A rung that lacks a predicted B1 strand is heavily penalized.

\section{4 $\alpha$-Helix Filter}

The secondary structure predictions output from PSIPRED is used to screen for $\alpha$ helices in the $\beta$-helical wrap. If the $\alpha$-helical content for a given wrap is more than $20 \%$ then the wrap is disqualified and the protein is rejected. The threshold value of $20 \%$ is determined from known $\beta$-helix proteins. The known $\beta$-helix proteins show an average $\alpha$-helical content of $8-14 \%$ [27]. All proteins that pass $\alpha$-helix filter and have a $\log$ odds score more than -35 are predicted as right-handed $\beta$-helix proteins. Similarly, if a putative rung shows presence of 4 or more amino acids to have a $\alpha$ helix secondary structure in PSIPRED results, then the rung is rejected.

\section{Results}

SVM-BetaPred performs at par in comparison to the state-of-art right-handed $\beta$-helix prediction methods. It recognizes the $\mathrm{R} \beta \mathrm{H}$-helix fold with $100 \%$ sensitivity and 99.6\% specificity as compared to BetaWrapPro [14] with $94.1 \%$ sensitivity and $99.4 \%$ specificity for known $\beta$-helix and non- $\beta$-helix proteins data set discussed in the materials and methods section. The method also succeeds in predicting the accurate rung position across the $\beta$-helix fold. It achieves $63.0 \%$ sensitivity and $68.1 \%$ specificity as compared to $58.0 \%$ sensitivity and $55.0 \%$ specificity for BetaWrapPro and $65.0 \%$ sensitivity and $46.3 \%$ specificity for BetaWrap on same set of known R $\beta H$ proteins. Homology search tools like PSI-BLAST and HMMER fail to recognize $\mathrm{R} \beta \mathrm{H}$ fold across superfamilies [1,14], thus results for SVM-BetaPred method are compared only with most accurate publicly available. A comparison of SVMBetaPred results is shown in Table 3. It should be noted that both BetaWrapPro and BetaWrap use HMMER to filter Left-handed $\beta$-helix and Leucine Rich Repeat proteins that appear as false positive predictions $[1,14]$. No such filter is used to the current SVM method.

The performance of dipeptide composition based SVM module is shown in Table 4. The performance of SVM modules was evaluated through 5-fold cross-validation. The dipeptide composition based SVM module (kernel $=\mathrm{RBF}, \gamma=18$ and $\mathrm{C}=900$ ) was able to predict rung with an average Q2 of $89.2 \%$ and $\mathrm{MCC}$ of 0.75 .

SVM-BetaPred predicts 498 proteins as RßH proteins. The pectate lyases and galacturonases are well represented among the predicted proteins. However, few of the high-scoring putative proteins that include ribosomal proteins, polymerase and other DNA replication enzymes; repeat proteins like WD repeat proteins and 
Table 3. Comparison of SVM-BetaPred, BetaWrapPro and BetaWrap results. Table denotes the percentage sensitivity $(\mathrm{Sn})$ and specificity $(\mathrm{Sp})$ obtained for known $\beta$-helices and rungs, dataset used for prediction (DB), number of proteins in dataset, number of predicted $\beta$-helix proteins (TP), false prediction in PDB minus dataset (FPmin) and PDB database (FP PDB) and incorrectly predicted folds (Folds). * denotes information in McDonnell et al [14].

\begin{tabular}{|c|c|c|c|c|c|c|c|c|c|c|}
\hline Tool & Sn & $\mathrm{Sp}$ & $\begin{array}{c}\text { Rung } \\
\text { Sn }\end{array}$ & $\begin{array}{c}\text { Rung } \\
\text { Sp }\end{array}$ & DB & $\begin{array}{c}\text { \# of } \\
\text { proteins }\end{array}$ & $\mathrm{TP}$ & $\begin{array}{l}\text { FP } \\
\text { min }\end{array}$ & $\begin{array}{c}\text { FP } \\
\text { PD } \\
\text { B }\end{array}$ & Folds \\
\hline $\begin{array}{l}\text { SVM } \\
- \\
\text { Beta } \\
\text { Pred }\end{array}$ & 100 & 99.6 & 63.0 & 68.1 & $\begin{array}{l}\text { Swiss- } \\
\text { Prot } 40 \\
\text { (Releas } \\
\text { e 51.2) }\end{array}$ & 67,835 & 498 & 18 & 42 & $\begin{array}{l}\beta-\text { san } \\
\text { dwich/ } \\
\text { barrel, } \\
\text { jelly } \\
\text { rolls, } \\
\text { repeat } \\
\text { protein }\end{array}$ \\
\hline $\begin{array}{l}\text { Beta } \\
\text { Wrap } \\
\text { Pro }\end{array}$ & 94.1 & 99.4 & 58.0 & 55.0 & $\begin{array}{l}\text { Swiss- } \\
\text { Prot } 40 \\
\text { (Releas } \\
\text { e 44.0) }\end{array}$ & 48,269 & 774 & 23 & 57 & $\begin{array}{l}\beta \text {-prop } \\
\text { eller/sa } \\
\text { ndwich } \\
/ \text { barrel, } \\
\alpha \text { helix, } \\
\alpha+\beta \text { pr } \\
\text { otein }\end{array}$ \\
\hline $\begin{array}{l}\text { Beta } \\
\text { Wrap }\end{array}$ & 93.8 & 98.5 & 65.0 & 46.3 & $\begin{array}{l}\text { Swiss- } \\
\text { Prot } \\
(39.6) \\
\text { and } \\
\text { TrEMB } \\
\text { L } \\
(14.11)\end{array}$ & $\begin{array}{c}595,89 \\
0\end{array}$ & -- & $\begin{array}{l}>30 \\
0 *\end{array}$ & - & $\begin{array}{l}\beta \text {-prop } \\
\text { eller/sa } \\
\text { ndwich } \\
/ \text { barrel, } \\
\alpha \text { helix, } \\
\alpha+\beta \text { pr } \\
\text { otein }\end{array}$ \\
\hline
\end{tabular}

Table 4. The predictive performance of dipeptide composition based SVM to predict rungs in protein sequences. The results were obtained by 5 -fold cross-validation.

\begin{tabular}{|c|c|c|c|c|}
\hline Data set & \multicolumn{4}{|c|}{ Prediction accuracy (\%) } \\
\hline & Sensitivity & Specificity & $\mathrm{Q}_{2}$ & MCC \\
\hline 1 & 64.2 & 100 & 88.7 & 0.74 \\
\hline 2 & 68.0 & 100 & 90.1 & 0.77 \\
\hline 3 & 63.3 & 100 & 88.8 & 0.73 \\
\hline 4 & 65.3 & 100 & 89.4 & 0.75 \\
\hline 5 & 63.3 & 100 & 88.8 & 0.73 \\
\hline
\end{tabular}

transmembrane proteins are likely false positives predictions from independent evidences and high homology to known structures. Table 5 available as supplementary material at http://www.scs.dauniv.ac.in/research.php lists all predicted right-handed ß-helix proteins, each ranked by p-value, Z-score, score, accession number, ID, source organism, description and the wrap position. 


\subsection{Recognition of Unknown Sequences}

SVM-BetaPred identifies a number of probable right-handed B-helices in the SW40 data set. Some of these include Pectate lyase precursor from Pseudomonas fluorescens (P0C1A7), Bacillus subtilis (P39116); Pectin lyase from Saccharomyces cerevisiae (P47180), Agrobacterium tumefaciens (P27644), Gibberella fujikuroi (Q07181), Actinidia chinensis (P35336) and Pseudomonas sp. (P58598); Chondroitinase from Pedobacter heparinus (Q46079); Carrageenase from Alteromonas carrageenovora (P43478) and Dextranase from Penicillium minioluteum (P48845). As reported earlier by Jenkins et al [5], there is a clear bias for the occurrence of RßH fold across the major group of organisms. Only $\sim 20 \%$ of the proteins predicted to contain $\mathrm{R} \beta \mathrm{H}$ fold belong to eukaryotes, furthermore only a few archeal and viral proteins show the presence of this fold. We found that proteins with $\mathrm{p}$-value $<0.65$ have a strong likelihood to display right-handed $\beta$-helix fold.

SVM-BetaPred successfully identifies newly solved $\beta$-helical protein hemoglobin protease (1wxr) from Escherichia coli with a p-value of 0.225 and $\beta$-roll subunit from C5 epimerase (2agm) from Azotobacter vinelandii with a p-value of 0.522 as $\mathrm{R} \beta \mathrm{H}$ protein. Interestingly both BetaWrapPro and BetaWrap fail to identify 2agm as righthanded $\beta$-helix.

\section{Discussions}

Machine learning methods like SVMs and neural networks are highly successful for residue state prediction where fixed window/pattern length is used [28]. In order to make optimal use of these techniques for protein structure prediction a fixed-length pattern must be generated. Amino acids composition that gives a fixed pattern length of 20 is commonly used by AI techniques for the classification of proteins. More information can be supplied by using by dipeptide composition. It gives a fixed pattern length of 400. Dipeptide composition has been widely used for the development of fold prediction methods [29] to achieve higher accuracies than that of amino acid composition based methods. A further step would be the use of tripeptide frequencies, however, AI techniques are unable to handle the noise due to the large number of input units and number of missing tripeptides in a protein. Thus, in this paper, we have constructed a SVM module on the basis of the dipeptide composition of $\beta$-helix proteins. This module is able to predict the rungs in a protein with overall accuracy of $90.1 \%$, as shown in Table 4.

To further improve prediction accuracy, a PSSM is developed to encapsulate more comprehensive information of $\beta$-helix proteins protein. This position weight matrix is used to score the rungs identified by SVM module and thus further enhance the $\beta$ helix fold prediction accuracy. The results confirmed that our approach is capable of capturing more information about super secondary structures like rungs that are vital to $\beta$-helix fold prediction. The method would complement the existing prediction tools for $\beta$-helix prediction. 


\section{References}

1. Bradley, P., Cowen, L., Menke, M., King, J., Berger, B.: BETAWRAP: Successful prediction of parallel beta helices from primary sequence reveals an association with many microbial pathogens. Proc. Natl. Acad. Sci. 98, 14819-14824 (2001)

2. Yoder, M.D., Jurnak, F.: The parallel $\beta$ helix and other coiled folds. FASEB J. 9(5), 335342 (1999)

3. Heffron, S., Moe, G., Sieber, V., Mengaud, J., Cossart, P., Vitali, J., Jurnak, F.: Sequence profile of the parallel beta helix in the pectate lyase superfamily. J. Struct. Biol. 122, 223 235 (1998)

4. Yonder, M., Keen, N., Jurnak, F.: New domain motif: The structure of pectate lyase C, a secreted plant virulence factor. Science 260(5113), 1503-1507 (1993)

5. Jenkins, J., Shevchik, V.E., Hugouvieux-Cotte-Pattat, N., Pickersgill, R.W.: The crystal structure of Pectate Lyase Pel9A from Erwinia chrysabthemi. J. Biol. Chem. 279(10), 9139-9145 (2004)

6. Iengar, P., Joshi, N.V., Padmanabhan, B.: Conformational and Sequence Signatures in $\beta$ Helix Proteins. Structure 14(3), 529-542 (2006)

7. Kreisberg, J.F., Betts, S.D., King, J.: ßeta-helix core packing within the triple-stranded oligomerization domain of P22 tailspike. Protein Sci. 9(12), 2338-2343 (2000)

8. Jenkins, J., Mayans, O., Pickersgill, R.: Structure and evolution of parallel helix proteins. Journal of Struct. Biol. 122, 236-246 (1998)

9. Leslie, C., Eskin, E., Noble, W.S.: The spectrum kernel: a string kernel for SVM protein classification. Pac. Symp. Biocomput., 564-575 (2002)

10. Cheng, J., Baldi, P.: A machine learning information retrieval approach to protein fold recognition. Bioinformatics 22(12), 1456-1463 (2006)

11. Murzin, A., Brenner, S., Hubbard, T., Chothia, C.: SCOP: a structural classification of proteins database for investigation of sequences and structures. J. Mol. Bio. 297, 536-540 (1995)

12. Altschul, S., Madden, T., Schaffer, A., Zhang, J., Zhang, Z., Miller, W., Lipman, L.: Gapped BLAST and PSI-BLAST: a new generation of protein database search programs. Nucleic Acids Res. 25, 3389-3402 (1997)

13. Eddy, S., Mitchison, G., Durbin, R.: Maximum discrimination hidden Markov models of sequence consensus. J. Comput. Biol. 2, 9-23 (1995)

14. McDonnell, A.V., Menke, M., Palmer, N., King, J., Cowen, L., Berger, B.: Prediction and comparative modeling of sequences directing beta-sheet proteins by profile wrapping. Proteins: Structure, Function, and Bioinformatics 63, 976-985 (2006)

15. Bairoch, A., Apweiler: The SWISS-PROT protein daabse and its supplement TrEMBL in 2000. Nucleic Acids Res. 28, 45-48 (2000)

16. Govaerts, C., Wille, H., Prusiner, S.B., Cohen, F.E.: Evidence for assembly of prions with left-handed beta-helices into trimers. Proc. Natl. Acad. Sci. USA 101(22), 8342-8347 (2004)

17. Li, W., Jaroszewski, L., Godzik, A.: Sequence clustering strategies improve remote homology recognitions while reducing search times. Protein Eng. 15(8), 643-649 (2002)

18. Zavaljevski, N., Stevens, F.J., Reifman, J.: Support vector machines with selective kernel scaling for protein classification and identification of key amino acid positions. Bioinformatics 18, 689-696 (2002)

19. Bhasin, M., Raghava, G.P.S.: ESLpred: SVM-based method for subcellular localization of eukaryotic proteins using dipeptide composition and PSI-BLAST. Nucleic Acids Research 32, W414-W419 (2004) 
20. Vapnik, V.N.: The Nature of Statistical Learning Theory. Springer, Heidelberg (1995)

21. Joachims, T.: Making large-scale SVM learning practical. In: Scholkopf, B., Burges, C., Smola, A. (eds.) Advances in Kernel Methods-Support Vector Learning. MIT Press, Cambridge, MA, London, England (1999)

22. Song, J., Burrage, K., Yuan, Z., Huber, T.: Prediction of cis/trans isomerization in proteins using PSI-BLAST profiles and secondary structure information. BMC Bioinformatics 7 , 124 (2006)

23. Matthews, B.W.: Comparison of predicted and observed secondary structure of T4 phage lysozyme. Biochim. Biophys. Acta. 405, 442-451 (1975)

24. Rice, P., Longden, I., Bleasby, A.: EMBOSS: The European Molecular Biology Open Software Suite. Trends in Genetics 16(6), 276-277 (2000)

25. Qiu, P., Cai, X.Y., Wang, L., Greene, J., Malcolm, B.: Hepatitis C virus whole genome position weight matrix and robust primer design. BMC Microbiology 2, 29 (2002)

26. Bryson, K., McGuffin, L.J., Marsden, R.L., Sodhi, J.S., Jones, D.T.: Protein structure prediction servers at University College London. Nucleic Acids Res. 1, 33 (2005)

27. Freiberg, A., Morona, R., Bosch, L., Baxa, U.: The Tailspike Protein of Shigella Phage Sf6. J. Biol. Chem. 278(3), 1542-1548 (2003)

28. Krogh, A., Riis, S.K.: Prediction of b sheets in protein. In: Touretzky, D.S., Mozer, M.C., Hasaselmo, M.E. (eds.) Advances in Neural Information Processing System 8, pp. 917 923. MIT Press, Cambridge, MA (1996)

29. Reczko, M., Bohr, H.: The DEF database of sequence based protein fold class prediction. Nucleic Acid Res. 22, 3616-3619 (1995) 